\title{
Prevalência e fatores associados à polifarmácia em idosos residentes em Rio Branco, Acre, Brasil: estudo transversal de base populacional, 2014*
}

\section{Prevalence of polypharmacy and associated factors in older adults living in Rio Branco, Acre, Brazil: cross-sectional population-based study, 2014}

\section{Prevalencia y factores asociados con la polifarmacia en residentes de adultos mayores en Rio Branco, Acre, Brasil: estudio transversal de base poblacional, 2014}

\author{
Gustavo Rodrigues de Rezende ${ }^{1}$ - (1) orcid.org/0000-0001-6145-6579 \\ Thatiana Lameira Maciel Amaral ${ }^{2}$ - @ orcid.org/0000-0002-9197-5633 \\ Cledir de Araújo Amaral ${ }^{3}$ - (1) orcid.org/0000-0002-7221-5364 \\ Maurício Teixeira Leite de Vasconcellos ${ }^{4}$ - @ orcid.org/0000-0003-1658-2589 \\ Gina Torres Rego Monteiro ${ }^{5}$ - (D orcid.org/0000-0002-9900-1825 \\ 'Universidade Federal da Bahia, Curso de Medicina, Salvador, BA, Brasil \\ ${ }^{2}$ Universidade Federal do Acre, Centro de Ciências da Saúde e do Desporto, Rio Branco, AC, Brasil \\ ${ }^{3}$ Instituto Federal do Acre, Rio Branco, AC, Brasil \\ ${ }^{4}$ Fundação Instituto Brasileiro de Geografia e Estatística, Escola Nacional de Ciências Estatísticas, Rio de Janeiro, RJ, Brasil \\ ${ }^{5}$ Instituto Oswaldo Cruz, Escola Nacional de Saúde Pública Sérgio Arouca, Programa de Pós-Graduação em Saúde, Rio de Janeiro, \\ RJ, Brasil
}

\section{Resumo}

Objetivo: Analisar a prevalência e os fatores associados à polifarmácia em idosos residentes em Rio Branco, Acre, Brasil, em 2014. Métodos: Estudo transversal de base populacional, obtido por amostragem complexa, com idosos. A polifarmácia foi definida como o uso concomitante de cinco ou mais fármacos. Resultados: A prevalência de polifarmácia foi de $14,9 \%$ ( $\mathrm{IC}_{95 \%}$ $11,8 ; 18,6)$, associada positivamente ao sexo feminino $\left(\mathrm{OR}=2,29-\mathrm{IC}_{95 \%} 1,41 ; 3,74\right)$, raça/cor da pele branca $(\mathrm{OR}=1,61-$ $\left.\mathrm{IC}_{95 \%} 1,10 ; 2,38\right)$, dependência $\left(\mathrm{OR}=1,65-\mathrm{IC}_{95 \%}, 1,05 ; 2,60\right)$, mudança de hábitos alimentares/uso de dieta $\left(\mathrm{OR}=1,66-\mathrm{IC}_{95 \%}\right.$ $1,16 ; 2,36)$, internação nos últimos 12 meses $\left(\mathrm{OR}=1,61-\mathrm{IC}_{05 \%}, 1,02 ; 2,53\right)$ e presença das seguintes morbidades autorreferidas: hipertensão arterial sistêmica $\left(\mathrm{OR}=2,40\right.$ - $\left.\mathrm{IC}_{95 \%} 1,33 ; 4,34\right)$, diabetes mellitus $\left(\mathrm{OR}=2,17-\mathrm{IC}_{95 \%} 1,23 ; 3,84\right)$, osteoporose $\left(\mathrm{OR}=2,92-\mathrm{IC}_{95 \%} 1,84 ; 4,64\right)$ e problemas cardíacos $\left(\mathrm{OR}=2,94-\mathrm{IC}_{95 \%} 1,90 ; 4,56\right)$. Conclusão: A polifarmácia em idosos encontrada neste estudo esteve associada às condições demográficas e de saúde.

Palavras-chave: Polimedicação; Idosos; Prevalência; Uso de Medicamentos; Estudos Transversais.

*Para sua realização, a pesquisa contou com o apoio financeiro do Conselho Nacional de Desenvolvimento Científico e Tecnológico (CNPq)/Ministério da Ciência, Tecnologia e Inovações (MCTI) - Processo nº 401081/2013-3 - e da Fundação de Amparo à Pesquisa do Acre (FAPAC) - Processo n 6068-14-0000029.

Endereço para correspondência:

Thatiana Lameira Maciel Amaral - Universidade Federal do Acre, Centro de Ciências da Saúde e do Desporto, Campus Universitário, BR 364, km 4, Distrito Industrial, Rio Branco, AC, Brasil, CEP:69920-900, Caixa postal: 500

E-mail: thatianalameira27@gmail.com 


\section{Introdução}

No Brasil, em 2015, a expectativa de vida ao nascer era de 75,4 anos e a proporção de idosos atingia 14,3\% da população, enquanto apenas 10 anos atrás, em 2005, essa proporção era de 9,8\%. Projeta-se para 2039 uma proporção de idosos de $24 \%$ da população brasileira. ${ }^{1}$

\section{A ocorrência da polifarmácia está relacionada com a maior demanda por medicamentos por idosos, e suas consequências incluem aumento da incidência de reações adversas a medicamentos, como a ocorrência de incontinência urinária, interações medicamentosas, de hospitalizações, do custo terapêutico e da mortalidade em idosos.}

Idosos, geralmente, apresentam multiplicidade de morbidades crônicas, um fato que torna o processo terapêutico complexo devido às modificações no corpo relacionadas com a própria idade, sexo, redução de habilidades cognitivas e sensoriais, além da maior dificuldade de adesão ao tratamento. ${ }^{2}$

A polifarmácia pode estar associada ao envelhecimento. ${ }^{3}$ A ocorrência da polifarmácia, definida como o uso concomitante de cinco ou mais fármacos, está relacionada com a maior demanda de medicamentos por idosos e suas consequências incluem aumento da incidência de reações adversas (aos medicamentos), a exemplo da incontinência urinária, interações medicamentosas e hospitalizações, além do custo terapêutico e da mortalidade. ${ }^{4}$

A prevalência de polifarmácia em idosos passou de 13,0 para 40,0\%, entre 1988 e 2010, nos Estados Unidos. ${ }^{5}$ No Brasil, no período de 2013 a 2014, essa prevalência foi de $18,0 \%$, associada a questões demográficas e morbidades. ${ }^{6}$

Estudos de base populacional que visam avaliar o perfil de utilização de medicamentos por idosos no Brasil são necessários, principalmente na Amazônia, devido a peculiaridades que incluem elevado analfabetismo e dificuldades de acesso a serviços de saúde na região. ${ }^{7}$ A alta prevalência de polifarmácia e a escassez de estudos sobre suas características e diferenças regionais motivaram a realização desta investigação, cujo objetivo foi analisar a prevalência e os fatores associados à polifarmácia em idosos de Rio Branco, Acre, Brasil.

\section{Métodos}

0 estudo consiste em um inquérito populacional realizado com idosos, a partir dos 60 anos, abrangendo as zonas urbana e rural de Rio Branco, capital do estado do Acre, no período de abril a setembro de 2014, com foco na estimativa da prevalência de polifarmácia.

Em 2010, Rio Branco dispunha de uma unidade territorial de $8.834,942 \mathrm{~km}^{2}$, com aproximadamente 21.620 pessoas idosas, em sua maioria residentes na zona urbana do município (91,8\%). No mesmo ano, Rio Branco alcançou o índice de desenvolvimento humano municipal (IDHM) de $0,727,{ }^{8}$ e a dimensão que mais contribuiu para esse feito foi a longevidade de seus cidadãos, com índice de 0,798 , seguida da renda $(0,729)$ e da educação $(0,661)$.

Foram elegíveis para 0 estudo todas as pessoas idosas a partir de 60 anos, de ambos os sexos, domiciliadas em Rio Branco; destas, foram excluídas todas as identificadas pelo entrevistador ou cujos familiares revelassem que apresentavam algum comprometimento cognitivo para sua comunicação e compreensão das questões.

Os participantes foram selecionados por amostragem probabilística em dois estágios: setor censitário e domicílio. Para a seleção dos setores censitários, empregou-se a probabilidade proporcional ao número de domicílios particulares registrados e disponíveis no Censo Demográfico 2010, realizado e publicado pelo Instituto Brasileiro de Geografia e Estatística (IBGE). Na seleção dos domicílios, partiu-se de amostragem sistemática com início aleatório e intervalos distintos em cada setor.

A seleção probabilística da amostra de idosos seguiu os procedimentos de amostragem por conglomerados, em dois estágios. Após a definição da amostra, definiu-se a seleção de 40 setores (do total de 338), como é usual em estudos epidemiológicos com amostragem baseada em dados censitários. A seleção de setores foi feita com probabilidade proporcional ao tamanho (PPT), para lidar com a diferença de tamanho entre os conglomerados. Logo, foram selecionados em cada setor, por amostragem sistemática, 73 domicílios, de 
forma independente, com intervalos iguais, sendo entrevistados todos os idosos residentes. Os detalhes sobre o plano amostral, cálculo e calibração dos pesos da amostra foram previamente publicados. ${ }^{9}$

As entrevistas foram realizadas no domicílio e incluíram a aplicação de questionário, estruturado em módulos temáticos, com as seguintes variáveis:

a) Socioeconômicas e demográficas

- Idade (em anos: 60-69; 70-79; 80 e mais);

- Sexo (masculino; feminino);

- Raça/cor da pele (branca; não branca [parda, preta, amarela e indígena]);

- Escolaridade (sem escolaridade; ensino fundamental completo; ensino médio completo; ensino superior);

- Estado conjugal (com companheiro; sem companheiro);

b) Comportamentais e de saúde (autorreferidas)

- Prática de atividade física (sim; não);

- Mudança de hábitos alimentares/dieta (sim; não);

- Doenças crônicas (hipertensão arterial; diabetes mellitus; artrite/artrose; osteoporose; problemas cardíacos; dislipidemia; depressão);

- Autoavaliação de saúde (muito boa/boa; regular; ruim/muito ruim);

- Internação nos últimos 12 meses (sim; não);

- Grau de independência (dependente; independente);

- Índice de massa corporal (IMC, em kg/m²: $\leq 27 \mathrm{~kg}$ / $\left.\mathrm{m}^{2} ;>27 \mathrm{~kg} / \mathrm{m}^{2}\right)$

- Sinais e sintomas de depressão (sim; não);

- Polifarmácia (sim; não).

Foram realizadas entrevistas para a coleta de dados. Os entrevistadores, habilitados e previamente treinados em curso específico, eram estudantes ou profissionais da área da saúde. Um manual com orientações, visando padronizar as entrevistas, foi elaborado e disponibilizado aos entrevistadores. A coleta de dados foi supervisionada pela coordenação do estudo.

Para investigar o grau de independência do idoso, foi empregada a escala de Atividades Instrumentais da Vida Diária (AIVD), composta por oito atividades: cuidar da casa; lavar a roupa; preparar a comida; ir às compras; usar o telefone; utilizar um meio de transporte; gerenciar 0 dinheiro; e gerenciar a medicação. ${ }^{10}$ Com base nessa escala de atividades e respectivas pontuações, os idosos que atingiram 27 pontos foram classificados como independentes, e aqueles com 26 pontos ou menos, dependentes.
Os sinais e sintomas de depressão foram investigados tendo como instrumento a Escala de Depressão Geriátrica. ${ }^{11}$ Esta escala apresenta pontuação de 0 a 15 , sendo considerados como casos sugestivos da doença aqueles com 6 pontos ou mais. Na análise do índice de massa corporal (IMC), levou-se em consideração os pontos de cortes da Nutrition Screening Initiative (NSI) para idosos com excesso de peso: IMC $>27 \mathrm{~kg} / \mathrm{m}^{2}{ }^{2}{ }^{12}$ Este ponto de corte leva em consideração as modificações corporais decorrentes do envelhecimento. ${ }^{12}$ Para determinação do IMC, foi realizada avaliação física, mediante a coleta de dados antropométricos do participante: peso e altura. 0 peso foi mensurado por uma balança digital Bal Gl $200 \mathrm{da}$ G-Tech ${ }^{\circledR}$, com resolução de 50 gramas, bem disposta (sobre base estável e plana), e a altura determinada por um estadiômetro portátil Sanny®, com resolução em milímetros, igualmente disposta, qual seja, sempre sobre uma superfície estável e plana.

0 uso de medicamentos atuais foi obtido por meio das seguintes perguntas:

"Utiliza alguma medicação? Se sim, quais os medicamentos, dose e frequência?"

Medicações de uso contínuo foram identificadas pela pergunta:

" $O(A) \operatorname{sr}($ a) utiliza algum desses medicamentos de forma contínua (um mês ou mais)?"

0 uso de medicamentos foi verificado pelo princípio ativo, dosagem e frequência, a partir da checagem da receita ou da embalagem de todos os medicamentos em uso no momento da avaliação. Para a categorização dos medicamentos, utilizou-se 0 Anatomical Therapeutic Chemical Code (ATC), adotado pela Organização Mundial da Saúde (OMS). ${ }^{13}$

A variável dependente avaliada foi a polifarmácia, definida como o 'uso concomitante de 5 ou mais fármacos' e dicotomizada em 'Sim' (uso de 5 ou mais medicamentos) e 'Não' (uso de 0 a 4 medicamentos). As variáveis independentes analisadas foram: sexo; idade; escolaridade; presença de morbidades autorreferidas; autoavaliação de saúde; grau de independência; sinais e sintomas sugestivos de depressão; obesidade; e prática de atividade física.

Para o controle da qualidade das informações, realizou-se um estudo-piloto com entrevistas e avaliações físicas de 30 idosos. ${ }^{14}$

Foi calculado o tamanho da amostra com base na prevalência de alteração da função renal entre 
idosos - 40\% - apontada em estudos internacionais, ${ }^{14}$ considerando-se a estimativa da população residente em Rio Branco no dia $1^{\circ}$ de julho de 2014, de 23.416 idosos, com nível de confiança de $95 \%$ e erro absoluto de 3 pontos percentuais. Como o plano amostral seguiu os procedimentos de amostragem complexa, foi utilizado efeito de desenho de 1,95 , resultando na estimativa de 1.020 idosos.

Realizaram-se análises descritivas dos dados, expressas em frequências absolutas e relativas, para caracterizar a população estudada. A análise das diferenças entre proporções baseou-se no teste qui-quadrado de Pearson.

A associação entre 0 uso de medicamentos e as variáveis independentes foi verificada por análise bruta e ajustada, mediante regressão logística, para facilitar sua comparação com os resultados de outros estudos, utilizando-se o modelo hierarquizado da medida de associação 'razão de chances': em inglês, odds ratio (OR). Adotou-se um processo de modelagem por blocos, em que, inicialmente, a polifarmácia foi ajustada pelas variáveis distais 'sexo' e 'raça/cor da pele'. No bloco intermediário, foram introduzidas as variáveis 'grau de independência', 'excesso de peso' e 'mudança de hábitos alimentares/dieta' e, finalmente, no bloco proximal, foram incorporadas as variáveis relacionadas às condições de saúde, visando controlar possíveis fatores de confundimento. Inicialmente, $o$ ajuste foi realizado dentro de cada nível do modelo, sendo incluídas as variáveis com valor de $\mathrm{p}<0,10$ na análise bivariada. No modelo final, foram mantidas as variáveis que alcançaram valor de $\mathrm{p}<0,05$. Adotou-se 0 nível de significância de $5 \%$, analisado pelo teste de Wald.

Foi utilizado o pacote estatístico SAS (versão 9.4). Todas as análises levaram em conta 0 efeito do desenho amostral e os pesos calibrados das observações; os resultados obtidos consideraram os pesos calibrados, visando sua extrapolação para a população por 'estimativa (n)'. Nesse sentido, foi utilizado o método de máxima pseudoverossimilhança (MPV), considerando-se os pesos amostrais e as informações estruturais do plano amostral. As inferências foram avaliadas pela estatística de Wald, com base no plano amostral, juntamente com a distribuição $\mathrm{F}$.

0 projeto do estudo foi aprovado pelo Comitê de Ética em Pesquisa com Seres Humanos da Universidade Federal do Acre (CEP/UFAC): Parecer n ${ }^{0} 518.531$, emitido em 30 de janeiro de 2014; Certificado de
Apresentação para Apreciação Ética (CAAE) $n^{\circ}$ 17543013.0.0000.5010. Os participantes do estudo assinaram o Termo de Consentimento Livre e Esclarecido, sendo garantido seu direito a recusa e sigilo dos dados informados.

\section{Resultados}

$\mathrm{Na}$ amostra de 1.016 entrevistados, houve um total de perdas e recusas de 59, que resultaram em uma população expandida de 23.416 idosos. A prevalência de polifarmácia nessa população foi de $14,9 \%\left(\mathrm{IC}_{95 \%} 11,8 ; 18,6\right)$.

A polifarmácia foi mais frequente entre as mulheres, indivíduos de raça/cor da pele branca, aqueles sem parceiro(a) e idosos mais velhos. Nos sedentários, ela foi de $14,3 \%$, e naqueles que realizaram mudança nos hábitos alimentares/dieta, de 21,9\% (Tabela 1).

A proporção encontrada de internação nos últimos 12 meses foi de 16,6\%. Naqueles em polifarmácia, essa prevalência foi de $23,7 \%$ (Tabela 1), sendo os principais motivos de internação a alteração na pressão arterial (23,7\%), seguida de problemas cardíacos $(17,9 \%)$, infecções $(14,1 \%)$, cirurgias $(13,7 \%)$ e acidente vascular encefálico (10,2\%).

Houve maior prevalência de polifarmácia entre os que relataram uma autoavaliação de saúde ruim e muito ruim, naqueles com sinais e sintomas de depressão, nos dependentes segundo o AIVD, entre os obesos; e naqueles com hipertensão arterial, diabetes mellitus, insônia, artrite/artrose, osteoporose, problemas cardíacos, dislipidemia e depressão, segundo autorrelato (Tabela 1).

$\mathrm{Na}$ análise ajustada por nível hierárquico, mantiveram significância estatística, no nível intermediário, as seguintes variáveis: sexo feminino; raça/cor da pele branca; dependência segundo AIVD; e mudança de hábitos alimentares/dieta. No terceiro modelo, do nível proximal, permaneceram as seguintes variáveis: raça/cor da pele branca; dependência segundo AIVD; internação nos últimos 12 meses; e morbidades autorreferidas - hipertensão arterial, diabetes mellitus, osteoporose e problemas cardíacos (Tabela 2).

\section{Discussão}

A prevalência de polifarmácia foi menor em idosos de Rio Branco, Acre, quando comparada 
Tabela 1 - Características dos participantes e prevalência de polifarmácia segundo variáveis sociodemográficas, hábitos de vida, condições de saúde e morbidades em idosos ( $n=1.016$ ), Rio Branco, Acre, 2014

\begin{tabular}{|c|c|c|c|c|c|}
\hline \multirow{2}{*}{ Variáveis } & \multicolumn{2}{|c|}{ Total } & \multicolumn{2}{|c|}{ Polifarmácia } & \multirow{2}{*}{$\mathrm{p}$-valor } \\
\hline & Estimativa (n) & $\%\left(\mathrm{IC}_{95 \%}{ }^{\mathrm{a}}\right)$ & Estimativa (n) & $\%\left(I C_{95 \%}{ }^{a}\right)$ & \\
\hline Sexo & & & & & 0,001 \\
\hline Masculino & 10.896 & $46,5(43,4 ; 49,7)$ & 1.040 & $9,5(6,2 ; 14,5)$ & \\
\hline Feminino & 12.520 & $53,5(50,3 ; 56,6)$ & 2.452 & $19,6(15,6 ; 24,3)$ & \\
\hline \multicolumn{2}{|l|}{ Idade (anos) } & & & & 0,303 \\
\hline $60-69$ & 13.394 & $57,2(54,7 ; 59,7)$ & 1.810 & $13,5(9,8 ; 18,4)$ & \\
\hline $70-79$ & 6.687 & $28,6(26,2 ; 31,1)$ & 1.054 & $15,8(11,4 ; 21,4)$ & \\
\hline$\geq 80$ & 3.335 & $14,2(12,2 ; 16,6)$ & 628 & $18,8(13,7 ; 25,4)$ & \\
\hline \multicolumn{2}{|l|}{ Raça/cor da pele } & & & & 0,017 \\
\hline Não branca & 5.614 & $76,0(71,8 ; 79,8)$ & 1.127 & $13,3(10,1 ; 17,3)$ & \\
\hline Branca & 17.802 & $24,0(20,2 ; 28,2)$ & 2.365 & $20,1(15,0 ; 26,3)$ & \\
\hline \multicolumn{2}{|l|}{ Estado conjugal } & & & & 0,721 \\
\hline Com companheiro & 9.086 & $39,1(35,7 ; 42,6)$ & 1.305 & $14,4(9,8 ; 20,6)$ & \\
\hline Sem companheiro & 14.172 & $60,9(57,4 ; 64,3)$ & 2.187 & $15,4(12,0 ; 19,6)$ & \\
\hline \multicolumn{2}{|l|}{ Escolaridade $^{c}$} & & & & 0,620 \\
\hline Sem escolaridade & 17.471 & $75,2(68,4 ; 81,0)$ & 2.612 & $15,0(11,5 ; 19,2)$ & \\
\hline Ensino fundamental & 1.963 & $8,5(6,2 ; 11,5)$ & 301 & $15,3(9,8 ; 23,2)$ & \\
\hline Ensino médio & 2.802 & $12,1(9,1 ; 15,8)$ & 380 & $13,5(8,1 ; 21,7)$ & \\
\hline Ensino superior & 987 & $4,2(2,7 ; 6,7)$ & 199 & $20,2(11,2 ; 33,6)$ & \\
\hline \multicolumn{2}{|c|}{ Prática de atividade físicac } & & & & 0,178 \\
\hline Sim & 3.480 & $14,9(11,9 ; 18,4)$ & 655 & $18,8(12,0 ; 28,3)$ & \\
\hline Não & 19.904 & $85,1(81,6 ; 88,1)$ & 2.837 & $14,3(11,4 ; 17,7)$ & \\
\hline \multicolumn{2}{|c|}{ Mudança de hábitos alimentares/dieta } & & & & $<0,001$ \\
\hline Sim & 7.372 & $35,6(32,4 ; 39,0)$ & 1.611 & $21,9(16,3 ; 28,7)$ & \\
\hline Não & 13.327 & $64,4(61,0 ; 67,6)$ & 1.395 & $10,5(7,8 ; 13,8)$ & \\
\hline \multicolumn{2}{|c|}{ Internação nos últimos 12 meses $^{c}$} & & & & 0,001 \\
\hline Sim & 3.746 & $16,6(20,0 ; 33,2)$ & 890 & $23,7(17,7 ; 31,1)$ & \\
\hline Não & 18.868 & $83,4(81,2 ; 85,5)$ & 2.526 & $13,4(10,3 ; 17,2)$ & \\
\hline \multicolumn{2}{|c|}{ Autoavaliação de saúde } & & & & 0,014 \\
\hline Muito bom/bom & 8.598 & $36,7(33,4 ; 40,1)$ & 856 & $10,0(6,7 ; 14,6)$ & \\
\hline Regular & 11.208 & $47,9(44,7 ; 51,0)$ & 1.770 & $15,8(11,3 ; 21,6)$ & \\
\hline Ruim/muito ruim & 3.610 & $15,4(12,9 ; 18,3)$ & 866 & $24,0(15,4 ; 35,3)$ & \\
\hline \multicolumn{2}{|c|}{ Sinais e sintomas de depressãoc } & & & & 0,002 \\
\hline Sim & 7.558 & $32,5(28,9 ; 36,4)$ & 1.462 & $19,3(15,0 ; 24,5)$ & \\
\hline Não & 15.666 & $67,5(63,6 ; 71,1)$ & 2.030 & $13,0(9,9 ; 16,9)$ & \\
\hline \multicolumn{2}{|c|}{ Grau de independência ${ }^{c}$} & & & & 0,003 \\
\hline Independente & 11.907 & $51,1(46,7 ; 55,5)$ & 1.350 & $11,3(7,9 ; 16,0)$ & \\
\hline Dependente & 11.402 & $48,9(44,5 ; 53,3)$ & 2.142 & $18,8(14,9 ; 23,4)$ & \\
\hline \multicolumn{2}{|c|}{ Índice de massa corporal (IMC: $\left.\mathrm{kg} / \mathrm{m}^{2}\right)^{\mathrm{c}}$} & & & & 0,027 \\
\hline$\leq 27$ & 11.321 & $51,4(46,2 ; 56,5)$ & 1.388 & $12,3(9,0 ; 16,6)$ & \\
\hline$>27$ & 10.714 & $48,6(43,5 ; 53,8)$ & 1.923 & $17,9(13,5 ; 23,4)$ & \\
\hline
\end{tabular}

a) $\mathrm{IC}_{95 \%}$ : intervalo de confiança de $95 \%$; b) Teste de Wald; c) Dados faltantes nessa variável devido a não resposta; d) Fibrilação, arritmia e insuficiência cardíaca. 
Continuação.

Tabela 1 - Características dos participantes e prevalência de polifarmácia segundo variáveis sociodemográficas, hábitos de vida, condições de saúde e morbidades em idosos ( $n=1.016$ ), Rio Branco, Acre, 2014

\begin{tabular}{|c|c|c|c|c|c|}
\hline \multirow{2}{*}{ Variáveis } & \multicolumn{2}{|c|}{ Total } & \multicolumn{2}{|c|}{ Polifarmácia } & \multirow{2}{*}{$\mathrm{p}$-valor } \\
\hline & Estimativa (n) & $\%\left(\mathbf{I C}_{95 \%}{ }^{\mathrm{a}}\right)$ & Estimativa (n) & $\%\left(\mathrm{IC}_{95 \%}{ }^{\mathrm{a}}\right)$ & \\
\hline \multicolumn{6}{|l|}{ Morbidades } \\
\hline Hipertensão arterial ${ }^{c}$ & & & & & $<0,001$ \\
\hline Sim & 13.958 & $59,8(56,3 ; 63,2)$ & 2.804 & $20,1(16,3 ; 24,6)$ & \\
\hline Não & 9.377 & $40,2(36,8 ; 43,7)$ & 646 & $6,9(4,3 ; 10,9)$ & \\
\hline Diabetes mellitus & & & & & $<0,001$ \\
\hline Sim & 4.060 & $17,7(15,3 ; 20,2)$ & 1.211 & $29,8(23,1 ; 37,6)$ & \\
\hline Não & 18.933 & $82,3(79,8 ; 84,7)$ & 2.260 & $11,9(9,1 ; 15,6)$ & \\
\hline Insôniac & & & & & 0,003 \\
\hline Sim & 8.039 & $34,4(31,6 ; 37,3)$ & 1.529 & $19,0(14,7 ; 24,3)$ & \\
\hline Não & 15.333 & $65,6(62,7 ; 68,4)$ & 1.963 & $12,8(9,8 ; 16,6)$ & \\
\hline Artrite/artrose & & & & & $<0,001$ \\
\hline Sim & 3.679 & $16,0(13,1 ; 19,5)$ & 1.034 & $28,1(21,0 ; 36,5)$ & \\
\hline Não & 19.251 & $84,0(80,5 ; 86,9)$ & 2.399 & $12,5(9,4 ; 16,4)$ & \\
\hline Osteoporose ${ }^{c}$ & & & & & $<0,001$ \\
\hline Sim & 3.453 & $15,1(12,7 ; 17,8)$ & 1.108 & $32,1(24,5 ; 40,8)$ & \\
\hline Não & 19.473 & $84,9(82,2 ; 87,3)$ & 2.300 & $11,8(9,0 ; 15,3)$ & \\
\hline Problemas cardíacos ${ }^{d}$ & & & & & $<0,001$ \\
\hline Sim & 2.432 & $10,4(8,4 ; 12,8)$ & 814 & $33,5(24,8 ; 43,4)$ & \\
\hline Não & 20.984 & $89,6(87,2 ; 91,6)$ & 2.678 & $12,8(10,0 ; 16,1)$ & \\
\hline Anemia $^{c}$ & & & & & 0,284 \\
\hline Sim & 1.726 & $7,5(5,9 ; 9,7)$ & 326 & $18,9(11,3 ; 29,8)$ & \\
\hline Não & 21.153 & $92,5(90,3 ; 94,1)$ & 3.082 & $14,6(11,5 ; 18,3)$ & \\
\hline Dislipidemia $^{c}$ & & & & & $<0,001$ \\
\hline Sim & 3.251 & $14,0(11,9 ; 16,5)$ & 877 & $27,0(19,3 ; 36,4)$ & \\
\hline Não & 19.897 & $86,0(83,5 ; 88,1)$ & 2.597 & $13,1(10,3 ; 16,4)$ & \\
\hline Depressãoc & & & & & 0,003 \\
\hline Sim & 2.736 & $11,9(9,6 ; 14,5)$ & 677 & $24,7(17,2 ; 34,3)$ & \\
\hline Não & 20.316 & $88,1(85,5 ; 90,4)$ & 2.783 & $13,7(10,7 ; 17,4)$ & \\
\hline Total & 23.416 & 100,0 & 3.492 & $14,9(11,8 ; 18,6)$ & \\
\hline
\end{tabular}

a) $\mathrm{IC}_{95 \mathrm{~g}}$ : intervalo de confiança de $95 \%$; b) Teste de Wald; c) Dados faltantes nessa variável devido a não resposta; d) Fibrilação, arritmia e insuficiência cardíaca.

Tabela 2 - Razão de chances (OR) e intervalo de confiança de $95 \%$ (IC ${ }_{95 \%}$ ) da polifarmácia entre idosos ( $\left.n=1.016\right)$, por variáveis, Rio Branco, Acre, 2014

\begin{tabular}{|c|c|c|c|c|}
\hline Variáveis & $\begin{array}{l}O R_{\text {bruta }}^{\mathrm{a}} \\
\left(\mathrm{IC}_{95 \%}^{\mathrm{b}}\right)\end{array}$ & p-valor & $\begin{array}{l}\mathrm{OR}^{\mathrm{a}}{ }_{\text {justada }} \\
\left(\mathrm{IC}_{95 \%} \mathrm{~b}^{\mathrm{b}}\right)\end{array}$ & p-valor \\
\hline \multicolumn{5}{|c|}{ Nível distal } \\
\hline Sexo & & 0,001 & & $0,00^{\mathrm{d}}$ \\
\hline Masculino & 1,00 & & 1,00 & \\
\hline Feminino & $2,31(1,42 ; 3,76)$ & & $2,29(1,41 ; 3,74)$ & \\
\hline
\end{tabular}

a) OR: odds ratio; b) IC $C_{95 \%}$ : intervalo de confiança de $95 \%$; c) Fibrilação, arritmia e insuficiência cardíaca; d) Nível distal (OR ajustada por sexo e raça/cor da pele); e) Nível intermediário (OR ajustada pelas variáveis significativas no modelo anterior açescidas de autoavaliaçăo de saúde, IMC, mudança de hábitos alimentares/dieta, sinais de sintomas de depressão e ser dependente); f) Nível proximal (OR ajustada pelas variáveis significativas no modelo anterior acrescidas de internação nos últimos 12 meses e morbidades autorreferidas). 
Continuação.

\section{Tabela 2 - Razão de chances (0R) e intervalo de confiança de $95 \%$ (IC $\left.{ }_{95 \%}\right)$ da polifarmácia entre idosos ( $\left.n=1.016\right)$,} por variáveis, Rio Branco, Acre, 2014

\begin{tabular}{|c|c|c|c|c|}
\hline Variáveis & $\begin{array}{l}O R_{\text {bruta }}^{\mathrm{a}} \\
\left(\mathrm{IC}_{95 \%}^{\mathrm{b}}\right)\end{array}$ & p-valor & $\begin{array}{l}\mathrm{OR}^{\mathrm{a}}{ }_{\text {jaustada }} \\
\left(\mathrm{IC} \mathrm{C}_{95 \%}{ }^{\mathrm{b}}\right)\end{array}$ & $\mathrm{p}$-valor \\
\hline Raça/cor da pele & & 0,017 & & $0,016^{d}$ \\
\hline Não branca & 1,00 & & 1,00 & \\
\hline Branca & $1,64(1,09 ; 2,45)$ & & $1,61(1,10 ; 2,38)$ & \\
\hline \multicolumn{5}{|c|}{ Nível intermediário } \\
\hline Grau de independência & & 0,003 & & $0,029^{e}$ \\
\hline Independente & 1,00 & & 1,00 & \\
\hline Dependente & $1,81(1,24 ; 2,64)$ & & $1,65(1,05 ; 2,60)$ & \\
\hline Índice de massa corporal (IMC: kg/m²) & & 0,027 & & $0,164^{\mathrm{e}}$ \\
\hline$\leq 27$ & 1,00 & & 1,00 & \\
\hline$>27$ & $1,57(1,06 ; 2,32)$ & & $1,37(0,87 ; 2,16)$ & \\
\hline Mudança de hábitos alimentares/dieta & & $<0,001$ & & $0,003^{e}$ \\
\hline Não & 1,00 & & 1,00 & \\
\hline Sim & $2,39(1,66 ; 3,45)$ & & $1,66(1,16 ; 2,36)$ & \\
\hline Sinais e sintomas de depressão & & 0,002 & & $0,144^{\mathrm{e}}$ \\
\hline Não & 1,00 & & 1,00 & \\
\hline Sim & & & $1,31(0,91 ; 1,90)$ & \\
\hline Autoavaliação de saúde & & 0,014 & & $0,240^{e}$ \\
\hline Muito bom/bom & 1,00 & & 1,00 & \\
\hline Regular & $1,70(1,03 ; 2,79)$ & & $1,46(0,82 ; 2,60)$ & \\
\hline Ruim e muito ruim & $2,85(1,38 ; 5,89)$ & & $2,06(0,85 ; 4,99)$ & \\
\hline \multicolumn{5}{|c|}{ Nível proximal } \\
\hline Internação nos últimos 12 meses & & 0,001 & & $0,041^{\dagger}$ \\
\hline Não & 1,00 & & 1,00 & \\
\hline Sim & $2,01(1,38 ; 2,94)$ & & $1,61(1,02 ; 2,53)$ & \\
\hline \multicolumn{5}{|l|}{ Morbidades autorreferidas } \\
\hline Hipertensão arterial & & $<0,001$ & & $0,005^{f}$ \\
\hline Não & 1,00 & & 1,00 & \\
\hline Sim & $3,39(2,16 ; 5,35)$ & & $2,40(1,33 ; 4,34)$ & \\
\hline Diabetes mellitus & & $<0,001$ & & $0,009^{f}$ \\
\hline Não & 1,00 & & 1,00 & \\
\hline Sim & $3,14(2,13 ; 4,62)$ & & $2,17(1,23 ; 3,84)$ & \\
\hline Insônia & & 0,003 & & $0,671^{f}$ \\
\hline Não & 1,00 & & 1,00 & \\
\hline Sim & $1,60(1,19 ; 2,15)$ & & $1,09(0,73 ; 1,64)$ & \\
\hline Artrite/artrose & & $<0,001$ & & $0,320^{f}$ \\
\hline Não & 1,00 & & 1,00 & \\
\hline Sim & $2,74(1,74 ; 4,32)$ & & $1,39(0,72 ; 2,69)$ & \\
\hline
\end{tabular}

a) OR: odds ratio; b) IC : intervalo de confiança de $95 \%$; c) Fibrilação, arritmia e insuficiência cardíaca; d) Nível distal (OR ajustada por sexo e raça/cor da pele);e) Nível intermediário (OR ajustada pelas variáveis significativas no modelo anterior acrescidas de autoavaliação de saúde, IMC, mudança de hábitos alimentares/dieta, sinais de sintomas de depressão e ser dependente);f) Nível proximal (OR ajustada pelas variáveis significativas no modelo anterior acrescidas de internação nos últimos 12 meses e morbidades autorreferidas). 
Continuação.

\section{Tabela 2 - Razão de chances (OR) e intervalo de confiança de 95\% ( $\left.\mathrm{IC}_{95 \%}\right)$ da polifarmácia entre idosos ( $\left.\mathrm{n}=1.016\right)$, por variáveis, Rio Branco, Acre, 2014}

\begin{tabular}{|c|c|c|c|c|}
\hline Variáveis & $\begin{array}{l}O \mathrm{R}_{\text {bruta }}^{\mathrm{a}} \\
\left(\mathrm{IC}_{95 \%}^{\mathrm{b}}\right)\end{array}$ & p-valor & $\begin{array}{l}O R^{a}{ }_{\text {ajustada }} \\
\left(\mathrm{IC}_{95 \%}{ }^{\mathrm{b}}\right)\end{array}$ & p-valor \\
\hline Osteoporose & & $<0,001$ & & $<0,001^{\dagger}$ \\
\hline Não & 1,00 & & 1,00 & \\
\hline Sim & $3,53(2,44 ; 5,10)$ & & $2,92(1,84 ; 4,64)$ & \\
\hline Problemas cardíacos ${ }^{c}$ & & $<0,001$ & & $<0,001^{\dagger}$ \\
\hline Não & 1,00 & & 1,00 & \\
\hline Sim & $3,44(2,30 ; 5,14)$ & & $2,94(1,90 ; 4,56)$ & \\
\hline Dislipidemia & & $<0,001$ & & $0,451^{f}$ \\
\hline Não & 1,00 & & 1,00 & \\
\hline Sim & $2,46(1,71 ; 3,53)$ & & $1,24(0,70 ; 2,19)$ & \\
\hline Depressão & & 0,003 & & $0,679^{f}$ \\
\hline Não & 1,00 & & 1,00 & \\
\hline Sim & $2,07(1,30 ; 3,30)$ & & $1,11(0,66 ; 1,88)$ & \\
\hline
\end{tabular}

a) OR:odds ratio; b) IC : intervalo de confiança de $95 \%$; c) Fibrilação, arritmia e insuficiência cardíaca; d) Nível distal (OR ajustada por sexo e raça/cor da pele); e) Nível intermediário (OR ajustada pelas variáveis significativas no modelo anterior acrescidas de autoavaliação de saúde, IMC, mudança de hábitos alimentares/dieta, sinais de sintomas de depressão e ser dependente); $f$ ) Nível proximal (OR ajustada pelas variáveis significativas no modelo anterior acrescidas de internação nos últimos 12 meses e morbidades autorreferidas).

às prevalências encontradas em outros estudos. ${ }^{5,6}$ Os fatores associados no nível distal foram o sexo feminino e ser de raça/cor da pele branca; no nível intermediário, os fatores que se mostraram associados foram a condição de dependência do idoso, segundo a avaliação das atividades da vida diária (AIVD), e a mudança de hábitos alimentares e/ou uso de dieta. No nível proximal, mostraram-se associadas à polifarmácia a internação nos últimos 12 meses e a presença das seguintes morbidades, autorreferidas: hipertensão arterial sistêmica, diabetes mellitus, osteoporose e problemas cardíacos.

A prevalência do presente estudo é inferior à observada em países desenvolvidos, como se observa ao se consultar um estudo conduzido em 2011, sobre uma população de 12.301 .537 idosos italianos com 65 anos ou mais, cuja prevalência de polifarmácia foi de $49,0 \%,{ }^{15}$ ou a prevalência de $39,0 \%$ encontrada nos Estados Unidos, em Pesquisa Nacional de Saúde e Nutrição (1988-2010) com uma amostra de 13.869 idosos $\geq 65$ anos. ${ }^{5}$ Trata-se de um valor maior que 0 encontrado por estudo populacional realizado em Cuiabá, no ano de 2012 (10,3\%), ${ }^{16}$ com 573 idosos; e entretanto, semelhante ao encontrado em um estudo realizado na região metropolitana de Belo Horizonte (14,3\%), ${ }^{17}$ este com 1.598 idosos, no ano de 2003. Contudo, a prevalência estimada neste trabalho sobre os idosos de Rio Branco foi inferior à resultante de uma pesquisa realizada em 2006, com indivíduos de 65 anos e mais residentes em São Paulo: 36,0\%. ${ }^{18}$ Tais informações indicam uma prevalência de polifarmácia no Brasil heterogênea, variando bastante segundo a região geográfica. Essa heterogeneidade pode decorrer das condições socioeconômicas e dos modelos de atenção à saúde adotados, assim como de fatores culturais, demográficos e do estado de saúde dos envolvidos. ${ }^{17}$

0 sexo feminino manteve associação com a polifarmácia em Rio Branco, conforme observado em trabalho conduzido no município de São Paulo, com 1.115 participantes na idade de 65 anos e mais. ${ }^{18} \mathrm{Tal}$ fato pode estar relacionado com o maior número de morbidades crônicas e, por conseguinte, maior procura por atendimento de saúde pelas mulheres. Aqui, cabe lembrar o que já foi reconhecido: elas são mais preocupadas com a condição de saúde e apresentam maior sobrevida que os homens. ${ }^{17}$

Pessoas consideradas dependentes referiram maior prevalência de polifarmácia que as mais independentes, haja vista o maior número de morbidades encontrar-se em pessoas dependentes, aumentando sua demanda por medicamentos. ${ }^{19}$ Estudo realizado na Irlanda, no período 2009-2011, com 3.499 idosos de 65 anos e mais, concluiu que o uso simultâneo de cinco ou mais medicamentos resultou em associação com as atividades instrumentais da vida diária 
$\left(0 \mathrm{R}=1,68-\mathrm{IC}_{95 \%}, 1,04 ; 2,70\right)$. A mesma pesquisa também observou a importância de ações preventivas para os fatores modificáveis associados às limitações funcionais. ${ }^{20}$ Deve-se ressaltar que, na maioria das vezes, o uso de medicamentos é apropriado. Porém, seu uso inadequado é uma realidade perigosa, sobretudo para a saúde funcional dos idosos.

Verificou-se, também, maior prevalência de polifarmácia em indivíduos que modificaram seus hábitos alimentares ou que fazem dieta regularmente. Essa informação pode ser explicada, ainda que parcialmente, pela orientação para mudança de hábitos alimentares ou prescrição de dieta específica, como resposta a um diagnóstico prévio de doenças relacionadas à obesidade. A perda de peso resulta em melhora da pressão arterial e dos níveis de glicemia ${ }^{21} \mathrm{e}$, assim, favorece 0 controle das doenças e o resultado esperado da terapia medicamentosa. No presente estudo, dada a limitação da temporalidade, não foi possível identificar o efeito dessa mudança na dieta.

A prevalência de internações hospitalares aumenta com o envelhecimento, em função da degeneração do estado de saúde, gerando maior demanda farmacoterapêutica. Ter sido internado nos últimos 12 meses apresentou associação positiva com a polifarmácia, como também relata outro estudo. ${ }^{18}$ As complicações cardiovasculares estão entre as principais causas de internação, segundo o presente estudo, e estão relacionadas com o envelhecimento, que favorece a arteriosclerose, diminuição da distensibilidade da aorta e das grandes artérias, comprometimento da condução cardíaca e redução na função barorreceptora. ${ }^{22}$

Em Rio Branco, entre as morbidades autorreferidas, a hipertensão arterial e os problemas cardíacos (fibrilação, arritmias e insuficiência cardíaca) foram mais prevalentes. Também foram abordados os efeitos da idade no sistema circulatório, observados anteriormente, assim como o diabetes mellitus e a osteoporose. Tais morbidades mantêm alta prevalência no país e demandam, na maioria dos casos, uso contínuo de medicamentos. ${ }^{23}$

0 diabetes mellitus é uma morbidade crônica, grave, podendo evoluir para complicações, sendo necessários medicamentos de uso contínuo para seu controle. ${ }^{24}$ Entre idosos, o autocontrole dos níveis glicêmicos de maneira adequada é pouco frequente, aumentando o risco de hipo ou hiperglicemia e, como consequência, complicações micro e macrovasculares que resultam em polifarmácia. Metas de melhoria da qualidade de vida e do aumento da expectativa de vida devem ser o foco de saúde para pacientes idosos diabéticos. Seu alcance é possível, pela manutenção do controle glicêmico, fato que exige um esforço coletivo de profissionais de saúde, familiares, cuidadores, e do próprio paciente..$^{25}$

Com 0 avançar da idade, a manifestação de problemas osteomusculares é recorrente e pode resultar em incapacidades e morbimortalidade. Outro estudo encontrou associação da osteoporose a maior consumo de medicamentos entre idosos, assim como o consumo abusivo de medicamentos também está associado ao aparecimento de osteoporose. ${ }^{26}$

Independentemente da morbidade, a escolha de fármacos para o tratamento e controle de doenças em idosos deve ser pautada na tomada de decisão compartilhada, devendo-se considerar a presença de comorbidades, polifarmácia, fragilidade física e potenciais efeitos adversos. Desta forma, garante-se aos pacientes idosos a consciência não só dos benefícios, mas também das possíveis complicações advindas do uso dos medicamentos indicados. A utilização de prontuários eletrônicos favorece os profissionais da saúde envolvidos na prescrição de medicamentos, na identificação de risco de eventos adversos e complicações de saúde, sendo importante a implementação de programas de gerenciamento de segurança de medicamentos $^{27}$ visando seu uso seguro, eficaz e adequado.

Torna-se imprescindível a eliminação de quaisquer dúvidas em relação aos regimes terapêuticos entre os idosos, cuidadores e familiares, para o que se mostra indispensável fornecer instruções precisas. Finalmente, os profissionais de saúde devem avaliar tanto a necessidade de tratar sintomas ou eventos adversos, quanto a de prescrever mais do que cinco medicamentos a um paciente; e sempre, solicitar e realizar uma verificação prévia dos medicamentos em uso, antes da prescrição de novos medicamentos. ${ }^{28}$

0 presente estudo de delineamento transversal de base populacional, com amostra de idosos não institucionalizados residentes em Rio Branco, Acre, revelou a prevalência de polifarmácia nessa população e seus fatores associados. Em virtude desse delineamento, cumpre reconhecer limitações inerentes à pesquisa. Inicialmente, não se pode atestar causalidade entre as variáveis estudadas. Ademais, os dados das morbidades foram obtidos por autorrelato, condição em que a falta 
de informação sobre o tempo transcorrido desde o diagnóstico pode ser um fator de confundimento para a polifarmácia; entretanto, a necessidade de autorrelato, buscando maior fidedignidade das informações, fez com que os pesquisadores optassem por esse critério de exclusão. Finalmente, a exclusão de indivíduos com comprometimento cognitivo e de comunicação pode ter subestimado a prevalência apresentada.

A prevalência de polifarmácia foi menor que a observada para o conjunto do país, com maior frequência entre as mulheres, pessoas que se autodeclararam de raça/cor da pele branca, idosos com dependência, que realizaram mudança de hábitos alimentares/uso de dieta, que foram internados nos últimos 12 meses e aqueles com hipertensão arterial sistêmica, diabetes mellitus, osteoporose e problemas cardíacos. Estes

\section{Referências}

1. Instituto Brasileiro de Geografia e Estatística - IBGE. Sinopse do Censo Demográfico 2010 [Internet]. Rio de Janeiro: IBGE; 2011 [citado 2011 dez 12]. Disponível em: http://biblioteca.ibge.gov.br/ visualizacao/livros/liv49230.pdf

2. Johansson T, Flamm M, Sönnichsen A, Schuler J. Interventions to reduce inappropriate polypharmacy: implications for research and practice. Maturitas [Internet]. 2017 Mar [cited 2021 Jan 6];97:668. Available from: https://doi.org/10.1016/j. maturitas.2016.12.007

3. Sirois C, Tannenbaum C, Gagnon M, Milhomme D, Émond V. Monitoring polypharmacy at the population level entails complex decisions: results of a survey of experts in geriatrics and pharmacotherapy. Drugs Ther Perspect [Internet]. 2016 Apr [cited 2021 Jan 6];32:257-64. Available from: https://doi. org/10.1007/s40267-016-0299-0

4. Maher RL, Hanlon J, Hajjar ER. Clinical consequences of polypharmacy in elderly. Expert Opin Drug Saf [Internet]. 2014 Jan [cited 2021 Jan 6];13(1):5765. Available from: https://dx.doi.org/10.1517\% 2F14740338.2013.827660

5. Charlesworth CJ, Smit E, Lee DS, Alramadhan F, Odden MC. Polypharmacy among adults aged 65 years and older in the United States: 1988-2010. J Gerontol A Biol Sci Med Sci [Internet]. 2015 Aug [cited 2021 Jan 6];70(8):989-95. Available from: https://doi. org/10.1093/gerona/glv013 segmentos sociais, identificados enquanto grupos mais vulneráveis à polifarmácia, necessitam de ações de farmacovigilância capazes de evitar efeitos da medicação indesejáveis para sua saúde.

\section{Contribuição dos autores}

Rezende GR, Amaral TLM, Amaral CA, Vasconcellos MTL e Monteiro GTR participaram da concepção e delineamento do estudo, análise e interpretação dos dados, redação do manuscrito e revisão de seu conteúdo. Todos os autores aprovaram a versão final do manuscrito e declaram-se responsáveis por todos os aspectos do trabalho, incluindo a garantia de sua precisão e integridade.

6. Ramos LR, Tavares NUL, Bertoldi AD, Farias MR, Oliveira MA, Luiza VL, et al. Polifarmácia e polimorbidade em idosos no Brasil: um desafio em saúde pública. Rev Saúde Pública [Internet]. 2016 [citado 2021 jan 6];50(Supl 2):9s. Disponível em: https://doi.org/10.1590/\$1518-8787.2016050006145

7. Guibu IA, Moraes JC, Guerra Junior AA, Costa EA, Acurcio FA, Costa KS, et al. Características principais dos usuários dos serviços de atenção primária à saúde no Brasil. Rev Saúde Pública [Internet]. 2017 [citado 2021 jan 6];51 (Suppl 2):17s. Disponível em: https://doi.org/10.11606/s15188787.2017051007070

8. Programa das Nações Unidas para o Desenvolvimento - PNUD. Atlas do Desenvolvimento Humano no Brasil 2013 [Internet]. [S.1.]: PNUD; 2013 [citado 2021 jan 6]. Disponível em: http://www.pnud.org.br/IDH/ Atlas2013.aspx?indiceAccordion=1\&li=li_Atlas 2013

9. Amaral TLM, Amaral CAA, Portela MC, Monteiro GTR, Vasconcellos MTL. Estudo das doenças crônicas (Edoc): aspectos metodológicos. Rev Saúde Pública [Internet]. 2019 jan [citado 2021 jan 6];53:8. Disponível em: https://doi.org/10.11606/s15188787.2019053000847

10. Lawton MP, Brody EM. Assessment of older people: self-maintaining and instrumental activities of daily living. Gerontologist [Internet]. 1969 Autumn [cited 2021 Jan 6];9(3):179-86. Available from: https://doi. org/10.1093/geront/9.3_Part_1.179 
11. Almeida OP, Almeida SA. Short versions of the geriatric depression scale: a study of their validity for the diagnosis of a major depressive episode according to ICD-10 and DSM-IV. Int J Geriatr Psychiatry [Internet]. 1999 Oct [cited 2021 Jan 6];14(10):85865. Available from: https://doi.org/10.1002/ (sici) 1099-1166(199910) 14:10\%3C858::aidgps35\%3E3.0.c0;2-8

12. Nutrition Screening Initiative. Nutrition interventions manual for professionals caring for older americans. Executive summary. Washington, DC: Nutrition Screening Initiative; 1992.

13. WHO Collaborating Centre for Drug Statistics Methodology. Guidelines for ATC classification and DDD assignment 2018 [Internet]. Oslo, Norway: WHO; 2017 [cited 2021 Jan 6]. Available from: https://www.drugsandalcohol.ie/29364/1/WHO\%20 Collaborating\%20Centre\%20for\%20Drug\%20 Statistics\%20Methodology.pdf

14. Stevens LA, Li S, Wang C, Huang C, Becker BN, Bomback AS, et al. Prevalence of CKD and comorbid illness in elderly patients in the United States: results from the Kidney Early Evaluation Program (KEEP). Am J Kidney Dis [Internet]. 2010 Mar [cited 2021 Jan 6];55(3 Suppl 2):S23-33. Available from: https:// doi.org/10.1053/j.ajkd.2009.09.035

15. Onder G, Bonassi S, Abbatecola AM, Folino-Gallo P, Lapi F, Marchionni N, et al. High prevalence of poor quality drug prescribing in older individuals: a nationwide report from the Italian Medicines Agency (AIFA). J Gerontol A Biol Sci Med Sci [Internet]. 2014 Apr [cited 2021 Jan 6];69(4):430-7. Available from: https://doi.org/10.1093/gerona/glt118

16. Almeida NA, Reiners AAO, Azevedo RCS, Silva AMC, Cardoso JDC, Souza LC. Prevalence of and factors associated with polypharmacy among elderly persons resident in the community. Rev Bras Geriatr Gerontol [Internet]. 2017 Jan-Feb [cited 2021 Jan 6];20(1):138-48. Available from: http://dx.doi. org/10.1590/1981-22562017020.160086

17. Loyola Filho AL, Uchoa E, Lima-Costa MF. Estudo epidemiológico de base populacional sobre uso de medicamentos entre idosos na Região Metropolitana de Belo Horizonte, Minas Gerais, Brasil. Cad Saúde Pública [Internet]. $2006 \mathrm{dez}$ [citado 2021 jan 6];22(12):2657-67. Disponível em: http://dx.doi. org/10.1590/S0102-311X2006001200015
18. Carvalho MFC, Romano-Lieber NS, BergstenMendes G, Secoli SR, Ribeiro E, Lebrão ML, et al. Polifarmácia entre idosos do Município de São Paulo - Estudo SABE. Rev Bras Epidemiol [Internet]. 2012 dez [citado 2021 jan 6];15(4):817-27. Disponível em: http://dx.doi.org/10.1590/\$1415790X2012000400013

19. Lima ALB, Espelt A, Lima KC, Bosque-Prous M. Activity limitation in elderly people in the European context of gender inequality: a multilevel approach. Ciênc Saúde Coletiva [Internet]. 2018 Sep [cited 2021 Jan 6];23(9):2991-3000. Available from: http://dx.doi. org/10.1590/1413-81232018239.20662016

20. Connolly D, Garvey J, McKee G. Factors associated with ADL/IADL disability in community dwelling older adults in the Irish longitudinal study on ageing (TILDA). Disabil Rehabil [Internet]. 2017 Apr [cited 2021 Jan 6];39(8):809-16. Available from: https:// doi.org/10.3109/09638288.2016.1161848 21.

21. Zhao Y, Zhang M, Luo X, Wang C, Li L, Zhang L, et al. Association of 6-year waist circumference gain and incident hypertension. Heart [Internet]. 2017 Sep [cited 2021 Jan 6];103(17):1347-52. Available from: https://doi.org/10.1136/heartjnl-2016-310760

22. Zaslavsky C, Gus I. Idoso: doença cardíaca e comorbidades. Arq Bras Cardiol [Internet]. 2002 [citado 2021 jan 6];79(6):635-9. Disponível em: http://publicacoes.cardiol.br/ abc/2002/7906/79060011.pdf

23. Tavares NUL, Costa KS, Mengue SS, Vieira MLFP, Malta DC, Silva Júnior JB. Uso de medicamentos para tratamento de doenças crônicas não transmissíveis no Brasil: resultados da Pesquisa Nacional de Saúde, 2013. Epidemiol Serv Saúde [Internet]. 2015 abr-jun [citado 2021 jan 6];24(2):315-23. Disponível em: http://dx.doi.org/10.5123/S167949742015000200014

24. Sociedade Brasileira de Diabetes -SBD. Diretrizes Sociedade Brasileira de Diabetes 2019-2020 [Internet]. São Paulo: Editora Clannad; 2019 [citado 2021 jan 6]. Disponível em: https://www. diabetes.org.br/profissionais/images/DIRETRIZESCOMPLETA-2019-2020.pdf

25. Atif M, Saleem Q, Babar ZU, Scahill S. Association between the vicious cycle of diabetes-associated complications and glycemic control among the elderly: a systematic review. Medicina (Kaunas) [Internet]. 2018 Oct [cited 2021 Jan 6];54(5):73. Available from: https://doi.org/10.3390/ medicina54050073 
26. O'Connell MB, Borgelt LM, Bowles SK, Vondracek SF. Drug-induced osteoporosis in the older adult. Aging Health [Internet]. 2010 Aug [cited 2021 Jan 6];6(4):501-18. Available from: https://doi. org/10.2217/ahe.10.42

27. Mair A, Fernandez-Llimos F, Alonso A, Harrison C, Hurding S, Kempen T, et al. Polypharmacy management by 2030: a patient safety challenge [Internet]. 2nd ed. Coimbra: SIMPATHY Consortium; 2017 [cited 2021 Jan 6]. Available from: https://

\section{Abstract}

Objective: To analyze polypharmacy prevalence and associated factors in older adults people living in Rio Branco, Acre, Brazil, in 2014. Methods: This was a cross-sectional population-based study using complex sampling with elderly people. Polypharmacy was defined as concomitant use of five or more medications. Results: Polypharmacy prevalence was 14.9\% (95\%IC 11.8;18.6), positively associated with females (OR=2.29 - 95\%CI $1.41 ; 3.74)$, white race/skin color $(O R=1.61-95 \% C I$ 1.10;2.38), dependence $(O R=1.65-95 \% C I 1.05 ; 2.60)$, change in eating habits/dieting (OR=1.66 - 95\%CI 1.16;2.36), hospitalization in the last 12 months $(O R=1.61-95 \% C I 1.02 ; 2.53)$ and presence of the following self-reported morbidities: systemic arterial bypertension $(O R=2.40-95 \%$ CI 1.33;4.34), diabetes mellitus (OR=2.17 - 95\%CI 1.23;3.84), osteoporosis $(O R=2.92-95 \% C I$ 1.84;4.64) and heart problems $(O R=2.94-95 \%$ CI 1.90;4.56). Conclusion: This study found that polypharmacy in older adults was associated with demographic and health conditions.

Keywords: Polypharmacy; Aged; Prevalence; Drug Utilization; Cross-sectional Studies. rgu-repository.worktribe.com/output/246840/ polypharmacy-management-by-2030-a-patient-safetychallenge

28. Alsuwaidan A, Almedlej N, Alsabti S, Daftardar O, Al Deaji F, Al Amri A, et al. A comprehensive overview of polypharmacy in elderly patients in Saudi Arabia. Geriatrics [Internet]. 2019 Jun [cited 2021 Jan 6];4(2):36. Available from: https://dx.doi. org/10.3390\%2Fgeriatrics 4020036

\section{Resumen}

Objetivo: Analizar la prevalencia y los factores asociados a la polifarmacia en adultos mayores residentes en Rio Branco, Acre, Brasil, en 2014. Métodos: Estudio poblacional transversal, verificación por muestreo complejo, con adultos mayores. La polifarmacia se definió como el uso concomitante de cinco o más fármacos. Resultados: La prevalencia de la polifarmacia fue de 14,9\% (IC95\% 11,8;18,6), asociada positivamente al sexo femenino (OR=2,29 - IC95\% $1,41 ; 3,74)$, raza/color de piel blanca $(O R=1,61-I C 95 \%$ $1,10 ; 2,38)$, dependencia $(O R=1,65$ - IC95\% 1,05;2,60), cambio en los hábitos alimentarios/dieta $(O R=1,66$ - IC95\% 1,16;2,36), hospitalización en los últimos 12 meses $(O R=1,61$ - IC95\% 1,02;2,53) y presencia de las siguientes morbilidades autoinformadas: hipertensión arterial sistémica (OR=2,40 - IC95\% 1,33;4,34), diabetes mellitus (OR=2,17 - IC95\% 1,23;3,84), osteoporosis (OR=2,92 - IC95\% 1,84;4,64) y problemas cardíacos (OR=2,94 - IC95\% 1,90;4,56). Conclusión: La polifarmacia en adultos mayores encontrada en este estudio se asoció a condiciones demográficas y de salud.

Palabras clave: Polifarmacia; Anciano; Prevalencia; Utilización de Medicamentos; Estudios Transversales.

Recebido em 09/06/2020

Aprovado em 24/11/2020 